\title{
Implicit Grasp Force Representation in Human Motor Cortical Recordings
}

\author{
John E. Downey 1,2,3,4, Jeffrey M. Weiss ${ }^{1,2,4}$, Sharlene N. Flesher ${ }^{1,2,4,5}$, \\ Zachary C. Thumser 6,7 , Paul D. Marasco ${ }^{6,8}$, Michael L. Boninger ${ }^{1,4,9}$, Robert A. Gaunt ${ }^{1,2,4}$ \\ and Jennifer L. Collinger ${ }^{1,2,4,9 *}$
}

${ }^{1}$ Department of Bioengineering, University of Pittsburgh, Pittsburgh, PA, United States, ${ }^{2}$ Center for the Neural Basis of Cognition, Pittsburgh, PA, United States, ${ }^{3}$ Department of Organismal Biology and Anatomy, University of Chicago, Chicago, IL, United States, ${ }^{4}$ Department of Physical Medicine and Rehabilitation, University of Pittsburgh, Pittsburgh, PA, United States, ${ }^{5}$ Department of Neurosurgery, Stanford University, Stanford, CA, United States, ${ }^{6}$ Laboratory for Bionic Integration, Department of Biomedical Engineering, Lerner Research Institute, Cleveland Clinic, Cleveland, $\mathrm{OH}$, United States, ${ }^{7}$ Research Service, Louis Stokes Cleveland VA Medical Center, Cleveland, OH, United States, ${ }^{8}$ Advanced Platform Technology Center of Excellence, Louis Stokes Cleveland VA Medical Center, Cleveland, OH, United States, ${ }^{9}$ VA Pittsburgh Healthcare System, Pittsburgh, PA, United States

\section{OPEN ACCESS}

Edited by:

Marc Slutzky,

Northwestern University,

United States

Reviewed by:

Gernot R. Müller-Putz,

Graz University of Technology, Austria

Aaron J. Suminski,

University of Wisconsin-Madison,

United States

*Correspondence:

Jennifer L. Collinger collinger@pitt.edu

Specialty section: This article was submitted to Neuroprosthetics,

a section of the journal

Frontiers in Neuroscience

Received: 02 May 2018 Accepted: 15 October 2018 Published: 31 October 2018

Citation:

Downey JE, Weiss JM,

Flesher SN, Thumser ZC,

Marasco PD, Boninger ML, Gaunt RA and Collinger JL (2018) Implicit Grasp

Force Representation in Human

Motor Cortical Recordings.

Front. Neurosci. 12:801.

doi: 10.3389/fnins.2018.00801
In order for brain-computer interface (BCl) systems to maximize functionality, users will need to be able to accurately modulate grasp force to avoid dropping heavy objects while also being able to handle fragile items. We present a case-study consisting of two experiments designed to identify whether intracortical recordings from the motor cortex of a person with tetraplegia could predict intended grasp force. In the first task, we were able classify neural responses to attempted grasps of four objects, each of which required similar grasp kinematics but different implicit grasp force targets, with 69\% accuracy. In the second task, the subject attempted to move a virtual robotic arm in space to grasp a simple virtual object. For each trial, the subject was asked to grasp the virtual object with the force appropriate for one of the four objects from the first experiment, with the goal of measuring an implicit representation of grasp force. While the subject knew the grasp force during all phases of the trial, accurate classification was only achieved during active grasping, not while the hand moved to, transported, or released the object. In both tasks, misclassifications were most often to the object with an adjacent force requirement. In addition to the implications for understanding the representation of grasp force in motor cortex, these results are a first step toward creating intelligent algorithms to help $\mathrm{BCl}$ users grasp and manipulate a variety of objects that will be encountered in daily life.

Clinical Trial Identifier: NCT01894802 https://clinicaltrials.gov/ct2/show/NCT018 94802.

Keywords: brain-computer interface, neuroprosthetics, motor cortex, intracortical, grasp force

\section{INTRODUCTION}

Brain-computer interfaces (BCI) have shown promise as assistive devices to restore a level of independence to people with tetraplegia (Collinger et al., 2012, 2013; Hochberg et al., 2012; Wodlinger et al., 2014; Blabe et al., 2015; Ajiboye et al., 2017). However, one significant limitation of the work to date is that control is limited to the kinematic domain where only the position or speed of the arm and hand are controlled (Collinger et al., 2012; Hochberg et al., 2012; Wodlinger et al., 2014). To achieve independence, users will need to modulate grasp force to maintain stable 
grasp postures and to handle objects that vary in weight, compliance, or fragility. Additionally, with the recent demonstration that intracortical microstimulation in somatosensory cortex can convey graded tactile percepts from many different locations on the hand (Flesher et al., 2016), it is now possible to provide feedback about grasp force through the BCI.

There is evidence from rats (Khorasani et al., 2016), non-human primates (Maier et al., 1993; Hepp-Reymond et al., 1999; Hendrix et al., 2009), and human subjects (Flint et al., 2014; Murphy et al., 2016) that grasp force-related information can be recorded from primary motor cortex (M1). Here we explore the representation of implicit grasp force from extracellular recordings in $\mathrm{M} 1$ of a single BCI user with tetraplegia, who is physically unable to generate overt grasping movements. We evaluate whether the visual presentation of objects of varying compliance and weight can elicit discriminable patterns of activity. We also investigate whether grasp force-related information is present during a multi-phase object transport task where no visual feedback about object identity is provided. During attempted grasping movements, M1 recordings clearly discriminated between objects with different force requirements, though this information was not present during reaching and object transport.

\section{MATERIALS AND METHODS}

This study was conducted under an Investigational Device Exemption from the U.S. Food and Drug Administration for this clinical trial (NCT01894802). The study was approved by the Institutional Review Boards at the University of Pittsburgh and the Space and Naval Warfare System Center Pacific. All procedures were conducted in accordance with the policies and guidelines associated with these approvals.

\section{Subject}

A 28 year-old male with C5 motor/C6 sensory ASIA B spinal cord injury provided written informed consent prior to participation. He had two $4 \times 4 \mathrm{~mm}$, 88-channel microelectrode arrays (Blackrock Microsystems, Salt Lake City, UT, United States) implanted in the hand and arm area of M1 in the left hemisphere (Locations shown in Figure 1C of Flesher et al., 2016). He also had two microelectrode arrays implanted into somatosensory cortex for stimulation that were not used in this experiment (Flesher et al., 2016).

\section{Neural Recording}

Extracellular potentials were hardware filtered between $0.3-7500 \mathrm{~Hz}$ and sampled at $30,000 \mathrm{~Hz}$, then digitally highpass filtered at $750 \mathrm{~Hz}$ with a first order Butterworth filter. At the beginning of each test session the spike threshold was set to -4.5 times the root-mean-square voltage on each channel. The number of threshold crossings on each channel were binned in $20 \mathrm{~ms}$ increments.

\section{Attempted Grasp vs. Object Observation}

The subject was asked to watch a television screen where images of four different objects were presented: a large marshmallow, a tomato, an orange, and a can of soup (Figure 1A). These objects were chosen in consultation with the subject to have varying levels of compliance and weight, which resulted in evenly spaced perceived levels of grasp force required to lift them. These objects were chosen, as opposed to explicitly named force levels, because previous work has shown that implicit force targets lead to more consistent grasp force exertion in able-bodied subjects (Thumser et al., 2018). The objects were approximately the same size, and the subject was instructed to attempt a 5-finger power grasp for all objects, even though his hand was paralyzed, in an attempt to keep grasp kinematics consistent. For all trials, the target object was shown for $4 \mathrm{~s}$, followed by $4 \mathrm{~s}$ of rest during which a fixation cross was displayed before the next trial started (Figure 1B). During attempted grasp trials, an audio cue was played $1.5 \mathrm{~s}$ after the object appeared to instruct the subject to attempt to grasp the object with the amount of force necessary to lift the object without crushing it. This strategy of attempting a movement even

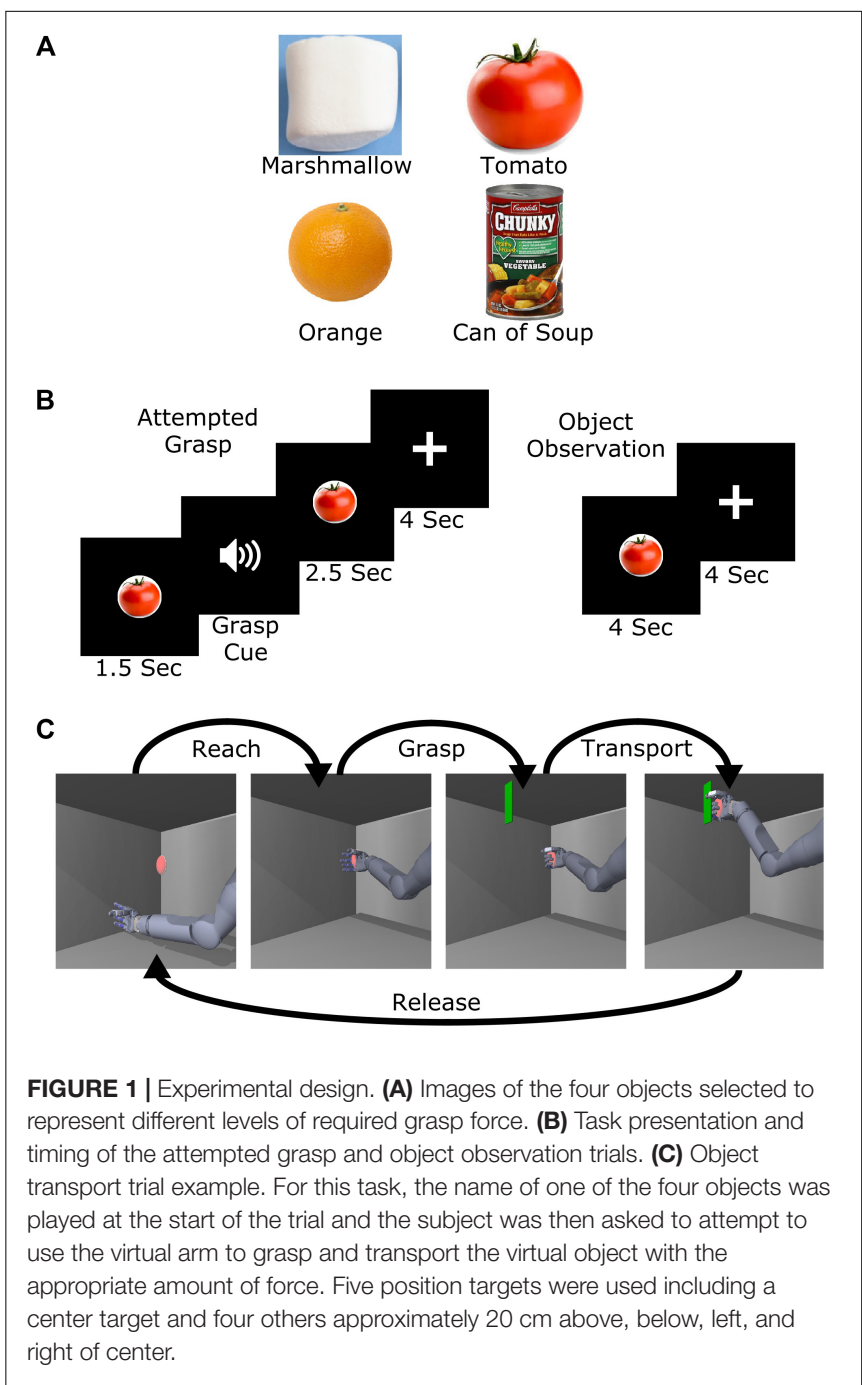


when no overt movement is generated is commonly used for BCI calibration (Taylor et al., 2002; Velliste et al., 2008; Collinger et al., 2012). During object observation trials, the subject was instructed to simply observe the objects without attempting to grasp. Across three test sessions (332, 372, and 378 days postimplant), 132 trials (33 per object) of both attempted grasp and object observation conditions were collected.

\section{Object Transport Task}

To study implicit grasp force representation in the context of whole arm movement, we adapted a virtual reality task (Wodlinger et al., 2014) where the arm moved in a twodimensional (2D) vertical plane with a single grasp dimension (flexion or extension of all fingers). The task consisted of a sequence of reaching to, grasping, transporting, and releasing a virtual object. The arm was moved automatically while the subject attempted to make the movements, as occurs during BCI calibration (Collinger et al., 2012; Wodlinger et al., 2014). At the start of each trial, one of the four objects (Figure 1A) was spoken as a computer-generated audio cue. Next, a red ellipsoid appeared at a target position (Figure 1C). Once the hand reached the ellipsoid (reach phase), an audio cue prompted the subject to attempt to grasp the object (grasp phase). The instructions to the subject were to attempt a 5-finger power grasp of the virtual object with the amount of force appropriate to hold the spoken object without letting it slip, even though his hand was paralyzed. A green plane then appeared and the subject attempted to move the ellipsoid to that location (transport phase). Finally, an audio cue instructed the subject to release the ellipsoid (release phase). The subject completed two sessions (374 and 576 days post-implant) of 99 trials each for a total of 198 trials.

\section{Classification of Object-Related Grasp Force}

To determine whether implicit grasp force was represented in M1 during attempted grasp and object observation, we performed naïve Bayes classification (Bishop et al., 2014; Perge et al., 2014) on $1 \mathrm{~s}$ of neural data starting $2 \mathrm{~s}$ after the start of the trial (Figure 1B). Thresholded channels with average firing rates higher than $2.5 \mathrm{~Hz}$ were used for classification; on average 125 channels were used for classification from each session. For attempted grasp and observation trials, the $1 \mathrm{~s}$ window started $2 \mathrm{~s}$ after the object was presented $(0.5 \mathrm{~s}$ after the grasp cue in grasp trials). This time period was selected based on a preliminary analysis showing chance-level classification prior to attempted grasp, with classification accuracy increasing during the $500 \mathrm{~ms}$ after the grasp cue. For cross-validation, we left out one random trial from each class (i.e., object type) during classifier training and then tested the classifier on the left-out trials, repeating until all trials had been left out. This process was repeated five times for each session to get multiple combinations of left-out trials. We estimated the $95 \%$ confidence intervals of chance level classification by shuffling the object labels and repeating the classification process 500 times.
We performed the same classification on neural data from the grasp and transport phases of the object transport task. We used 157 channels for classification in session 1 and 131 channels in session 2. For the grasp phase, the last $1 \mathrm{~s}$ of data were used for classification. For the transport phase, we selected $1 \mathrm{~s}$ of active reaching, which ended $500 \mathrm{~ms}$ before the end of the phase.

We further examined how classification accuracy evolved over the duration of object transport trials using a sliding window of neural data. Since the reach and transport phases were different lengths across trials, data were aligned to the grasp and release cues. A $500 \mathrm{~ms}$ trailing window with $100 \mathrm{~ms}$ step sizes was used to classify grasp force level for $1.6 \mathrm{~s}$ before and $0.8 \mathrm{~s}$ after the grasp and release cues. Confidence intervals on chance level performance were estimated for each classification window using shuffled object labels as described above.

\section{RESULTS}

\section{Object Observation vs. Attempted Grasp}

The subject, who was unable to move his own hand voluntarily, was asked to attempt to grasp visually presented objects (marshmallow, tomato, orange, or can of soup) with an appropriate amount of force to lift them. We could predict with $69 \%$ accuracy which object he was attempting to grasp using $1 \mathrm{~s}$ of neural data starting $500 \mathrm{~ms}$ after the grasp cue. This was significantly better than the chance rate of $25 \pm 9 \%$ (95\% confidence interval). Additionally, 97\% of misclassified trials were predicted to be an object with an adjacent implicit grasp force level (95\% confidence bounds on chance: $51 \pm 10 \%$, Figure 2A). When the subject simply observed the objects, but did not attempt to grasp, classification accuracy was $29 \%$, which was no better than chance ( $95 \%$ confidence bounds on chance: $25 \pm 9 \%)$.

\section{Object Transport Task}

We also examined whether grasp force information could be classified in a more complex task where the subject attempted reaching to, grasping, and transporting an object with an appropriate amount of force. During the grasp phase, object type could be predicted with $51 \%$ accuracy and $73 \%$ of misclassifications fell within one force level of the target (Figure 2B). However, classification accuracy during the transport phase was only $25 \%$, which was no better than chance. During transport, only $56 \%$ of misclassifications were within one force level of the target.

A sliding window classifier revealed the time course of classification accuracy. This analysis used $500 \mathrm{~ms}$ of neural data as compared to $1 \mathrm{~s}$ for the classification results presented above. During the reach phase, object identity could only be classified at chance level (26\%). However, over the course of the grasp phase, classification accuracy improved from $34 \%$ at grasp phase onset to $47 \% 800 \mathrm{~ms}$ into the grasp phase (95\% confidence bounds on chance: $25 \pm 7 \%$, Figure 2C). During the transport phase, classification accuracy returned to chance level (27\%). The subject confirmed that he continued to attempt to grasp the object with the appropriate 


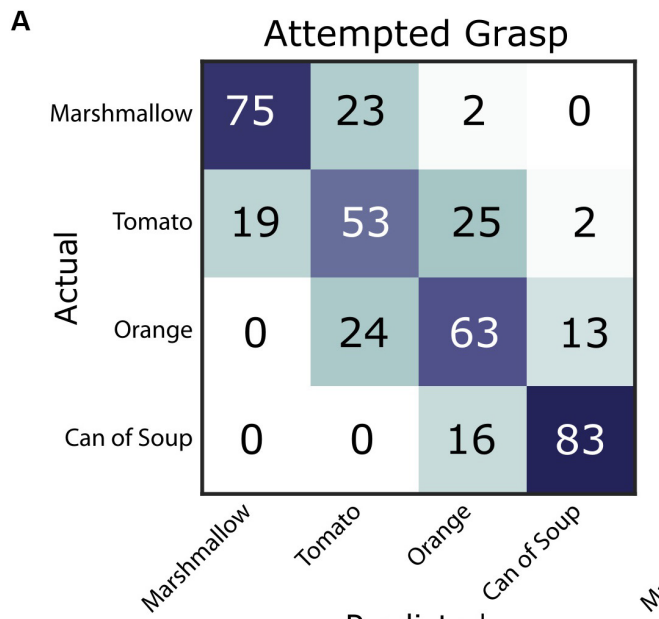

Predicted

B

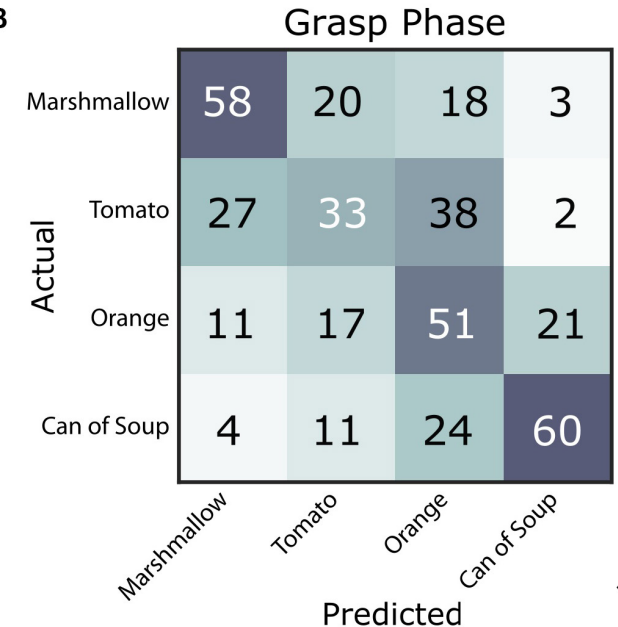

Object Observation

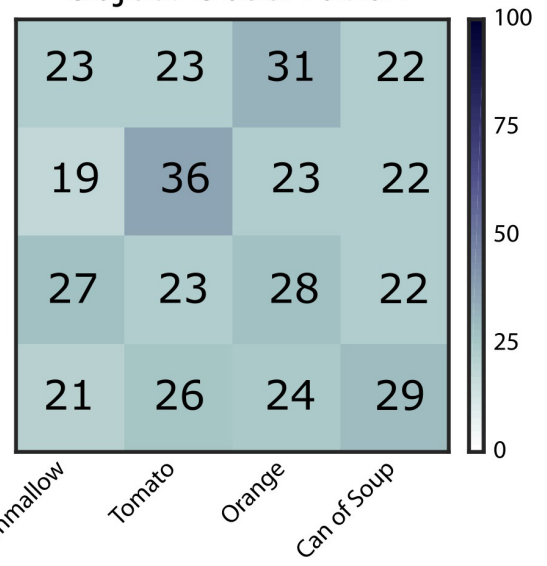

Predicted

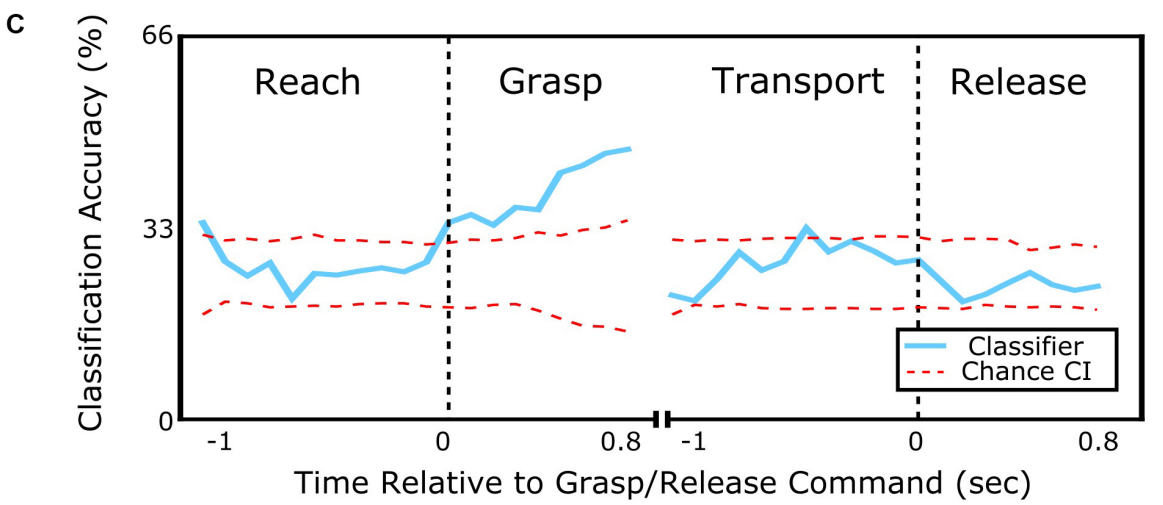

FIGURE 2 | Classification of object identity for objects of various compliances and grasp force requirements. (A) Confusion matrices showing classification accuracy for each object from the attempted grasp vs. object observation task. Each row shows the percentage of times the corresponding object was predicted to be each of the four possible objects (numbers may not add to 100 due to rounding). On the left, classification of the presented object is accurate (69\% success) when the subject is attempting to grasp it. Errors are typically to adjacent objects in terms of compliance and required grasp force. On the right, classification of the presented object when the subject simply observes the object is within chance and classification errors are randomly distributed. (B) Confusion matrices showing classification accuracy from the grasp and transport phases of the object transport task. On the left, classification is accurate (50\% success) during the grasp phase with errors typically to an adjacent object in terms of required grasp force. On the right, classification during the transport phase is near chance and classification errors are randomly distributed. (C) Classification accuracy computed for the object transport task, shown as the blue line aligned to the cue to grasp (left) and release (right). The red dotted lines bound the $95 \%$ confidence interval on chance performance. Classification is above chance during the grasp phase, but is generally within chance level for the other phases of movement. 
force during the transport phase. Finally, during the release phase, average classification accuracy remained at chance level (23\%).

\section{DISCUSSION}

Here we show that implicit grasp force is represented in M1 during attempted grasp, but not during observation, of objects of varying compliance, weight and texture. Importantly, implicit force was not well-represented in M1 during periods where the force was maintained, such as transport, or when the object was released. While previous studies identified grasp force information in EEG signals (Rearick et al., 2001; Murguialday et al., 2007; Paek et al., 2015; Wang et al., 2017) and intracortical field potentials of motor-intact subjects (Flint et al., 2014; Murphy et al., 2016), here we show that this information is present in multi-unit recordings of a subject with tetraplegia. To identify information about implicit grasp force in a subject who could not generate grasp forces, we asked him to attempt to grasp objects that he was familiar with grasping before his injury. Our result that M1 contains implicit information about grasp force aligns well with data from able-bodied subjects showing that the use of everyday objects as implicit grasp force cues led to a more consistent separation of executed grasp forces during the initial dynamic phase of the grasp as compared to trying to match a visual force target (Thumser et al., 2018).

We observed that implicit grasp force was not wellrepresented in M1 during object transport, which is consistent with a prior report that grasp force encoding is context dependent (Hepp-Reymond et al., 1999). However, it is inconsistent with reports that more neurons were modulated during static grasp force exertion than during the initial application of grasp force (Mason et al., 2002). One possibly important difference between the Mason et al. (2002) study and ours is that their work did not include object transport while grasping, which could change the context of the coding in M1. Another difference is that Mason et al. (2002) used single electrode recordings in multiple sites of a large window spanning M1 and dorsal premotor cortex compared to our microelectrode array recordings from a smaller, fixed location. This difference could have resulted in Mason et al. (2002) recording from a more heterogenous population of neurons, including corticomotor neurons that make monosynaptic connections to the motoneurons innervating the muscles in the hand and forearm (Rathelot and Strick, 2009). Identifying the cause of this change in coding between movement phases will require additional experiments.

Our study design required the participant to attempt to grasp familiar objects in order to elicit an implicit representation of grasp force even though no overt movement was performed due to his paralysis. The subject was instructed to use consistent grasp kinematics to pick up the objects without deforming them, however, there was no way to measure his attempted kinematics because he could not move his own hand. Because the movements were performed covertly, there are limitations to the interpretation of the results. We were unable to measure the impact that differences in the visualized kinematics or object properties, such as texture or compliance, had on the neural activity. The objects were chosen to be equally spaced within the subject's reported implicit force range. During attempted grasping of these objects, nearly all classification errors were to an object that was directly adjacent to the target object on the implicit force spectrum. This is despite the fact that the two cylindrical objects that were most similar in shape (large marshmallow and can of soup) were at opposite ends of the force spectrum. Previous work by Schaffelhofer et al. (2015) showed that cylindrical objects of differing sizes, like our marshmallow and can of soup, were more likely to be confused with each other by a classifier using recordings from M1 than with spheres, like our tomato and orange, of any size when primates planned and executed movements to a large variety of different sized and shaped objects. The systematic classification prediction error in our results suggests that implicit grasp force is represented in M1 in an intuitive way.

The familiar objects used in this study differ in terms of visual appearance and expected sensory consequences (e.g., texture and compliance). If the visual and sensory properties of the objects were the primary driver of neural activity, we would have expected misclassification errors to be more randomly distributed. The decision to use familiar objects was motivated by previous work in able-bodied subjects that found that grasp forces were applied more consistently when presented with familiar objects as compared to being instructed to hit explicit force targets on a single object (Thumser et al., 2018). It is also important to note that M1 activity was modulated during the grasp phase of the object transport task, even though the object being grasped remained visually the same for all trials (i.e., a red ellipsoid). This provides additional evidence that planning for slight differences in grasp kinematics or finger placement is not a primary driver of classification accuracy.

We leveraged the implicit neural representation of grasp force for familiar objects to identify grasp force information in M1. The classification accuracies reported here, while above chance, will need to improve to provide reliable $\mathrm{BCI}$ control and give the user continuous control over grasp force. Additionally, the $\mathrm{BCI}$ will need information about grasp kinematics or state (e.g., open/close). Further work is necessary to optimally extract this information and determine whether this representation of grasp force generalizes well to other objects. Based on this work, we believe that decoders will need to include intelligent algorithms that can recognize when grasp force information is available in M1 and then exert that force through the prosthesis at the appropriate times during object manipulation. Similarly, for BCIs that aim to restore native limb movement through muscle stimulation, one could modulate stimulation intensity based on intended grasp force (Bouton et al., 2016; Ajiboye et al., 2017). Since this study was conducted while the subject was at rest with only visual feedback of the task, it will also be important to determine if feedback provided through restored somatosensory pathways changes the internal representation of grasp force (Flesher et al., 2016).

We have presented evidence, in a case-study, that attempted grasping of familiar objects elicits patterns of activity in M1 that 
are informative about implicit grasp force representations. Forcerelated activity was most robust during periods of active grasping and not during periods of static force application.

\section{AUTHOR CONTRIBUTIONS}

JD, ZT, PM, MB, RG, and JC contributed to the design of the study. JD and JW implemented the experimental paradigms. JD, JW, and SF ran experiments. JD analyzed the data. JD, SF, RG, and JC interpreted the results. All authors contributed to, and approved, the manuscript.

\section{FUNDING}

This material is based upon work supported by the Defense Advanced Research Projects Agency (DARPA) and Space and Naval Warfare Systems Center Pacific (SSC Pacific) under Contract No. N66001-16-C-4051, the Revolutionizing Prosthetics program under Contract No. N66001-10-C-4056 and

\section{REFERENCES}

Ajiboye, A. B., Willett, F. R., Young, D. R., Memberg, W. D., Murphy, B. A., Miller, J. P., et al. (2017). Restoration of reaching and grasping movements through brain-controlled muscle stimulation in a person with tetraplegia: a proof-ofconcept demonstration. Lancet 389, 1821-1830. doi: 10.1016/S0140-6736(17) 30601-3

Bishop, W., Chestek, C. A., Gilja, V., Nuyujukian, P., Foster, J. D., Ryu, S. I., et al. (2014). Self-recalibrating classifiers for intracortical brain-computer interfaces. J. Neural Eng. 11:26001. doi: 10.1088/1741-2560/11/2/026001

Blabe, C. H., Gilja, V., Chestek, C. A., Shenoy, K. V., Anderson, K. D., and Henderson, J. M. (2015). Assessment of brain-machine interfaces from the perspective of people with paralysis. J. Neural Eng. 12:043002. doi: 10.1088/ 1741-2560/12/4/043002

Bouton, C. E., Shaikhouni, A., Annetta, N. V., Bockbrader, M. A., Friedenberg, D. A., Nielson, D. M., et al. (2016). Restoring cortical control of functional movement in a human with quadriplegia. Nature 533, 247-250. doi: 10.1038/ nature 17435

Collinger, J. L., Boninger, M. L., Bruns, T. M., Curley, K., Wang, W., and Weber, D. J. (2013). Functional priorities, assistive technology, and brain-computer interfaces after spinal cord injury. J. Rehabil. Res. Dev. 50, 145-160.

Collinger, J. L., Wodlinger, B., Downey, J. E., Wang, W., Tyler-Kabara, E. C., Weber, D. J., et al. (2012). High-performance neuroprosthetic control by an individual with tetraplegia. Lancet 6736, 1-8. doi: 10.1016/S0140-6736(12)61816-9

Flesher, S. N., Collinger, J. L., Foldes, S. T., Weiss, J. M., Downey, J. E., Tyler-Kabara, E. C., et al. (2016). Intracortical microstimulation of human somatosensory cortex. Sci. Transl. Med. 8, 1-11. doi: 10.1126/scitranslmed.aaf8083

Flint, R. D., Wang, P. T., Wright, Z. A., King, C. E., Krucoff, M. O., Schuele, S. U., et al. (2014). Extracting kinetic information from human motor cortical signals. Neuroimage 101, 695-703. doi: 10.1016/j.neuroimage.2014.07.049

Hendrix, C. M., Mason, C. R., and Ebner, T. J. (2009). Signaling of grasp dimension and grasp force in dorsal premotor cortex and primary motor cortex neurons during reach to grasp in the monkey. J. Neurophysiol. 102, 132-145. doi: 10.1152/jn.00016.2009

Hepp-Reymond, M.-C., Kirkpatrick-Tanner, M., Gabernet, L., Qi, H.-X., and Weber, B. (1999). Context-dependent force coding in motor and premotor cortical areas. Exp. Brain Res. 128, 123-133. doi: 10.1007/s0022100 50827

Hochberg, L. R., Bacher, D., Jarosiewicz, B., Masse, N. Y., Simeral, J. D., Vogel, J., et al. (2012). Reach and grasp by people with tetraplegia using a
Hand Proprioception and Touch under Contract No. N6600115-C-4015.

\section{ACKNOWLEDGMENTS}

We thank Nathan Copeland for his extraordinary commitment and effort in relation to this study and insightful discussions with the study team; Debbie Harrington (Physical Medicine and Rehabilitation) for regulatory management of the study; the University of Pittsburgh Clinical and Translational Science Institute and the Office of Investigator-Sponsored Investigational New Drugs and Investigational Device Exemption support for assistance with protocol development and regulatory reporting and compliance; the volunteer members of the DSMB for their continued monitoring of this study; and Blackrock Microsystems (Salt Lake City, UT, United States) for technical support in relation to this project. The views, opinions, and/or findings contained in this article are those of the authors and should not be interpreted as representing the official views or policies of the Department of Veterans Affairs, Department of Defense, or US Government.

neurally controlled robotic arm. Nature 485, 372-375. doi: 10.1038/nature1 1076

Khorasani, A., Heydari Beni, N., Shalchyan, V., and Daliri, M. R. (2016). Continuous force decoding from local field potentials of the primary motor cortex in freely moving rats. Sci. Rep. 6, 1-10. doi: 10.1038/srep35238

Maier, M. A., Bennett, K. M., Hepp-Reymond, M. -C., and Lemon, R. N. (1993). Contribution of the monkey corticomotoneuronal system to the control of force in precision grip. J. Neurophysiol. 69, 772-85. doi: 10.1152/jn.1993.69. 3.772

Mason, C. R., Gomez, J. E., and Ebner, T. J. (2002). Primary motor cortex neuronal discharge during reach-to-grasp: controlling the hand as a unit. Arch. Ital. Biol. 140, 229-236.

Murguialday, A. R., Aggarwal, V., Chatterjee, A., Cho, Y., Rasmussen, R., O'Rourke, B., et al. (2007). "Brain-computer interface for a prosthetic hand using local machine control and haptic feedback," in Proceedings of the 2007 IEEE 10th Internationl Conference Rehabilitation Robotics (Noordwijk: IEEE), 609-613. doi: 10.1109/ICORR.2007.4428487

Murphy, B. A., Miller, J. P., Gunalan, K., and Bolu Ajiboye, A. (2016). Contributions of subsurface cortical modulations to discrimination of executed and imagined grasp forces through stereoelectroencephalography. PLoS One 11:e0150359. doi: 10.1371/journal.pone.0150359

Paek, A. Y., Gailey, A., Parikh, P., Santello, M., and Contreras-Vidal, J. (2015). "Predicting hand forces from scalp electroencephalography during isometric force production and object grasping," in Proceedings of the Annual International Conference IEEE Engineering Medicine and Biology Society EMBS 2015-Novem, Philadelphia, PA, 7570-7573. doi: 10.1109/EMBC.2015.732 0144

Perge, J. A., Zhang, S., Malik, W. Q., Homer, M. L., Cash, S., Friehs, G., et al. (2014). Reliability of directional information in unsorted spikes and local field potentials recorded in human motor cortex. J. Neural Eng. 11:046007. doi: 10.1088/1741-2560/11/4/046007

Rathelot, J.-A., and Strick, P. L. (2009). Subdivisions of primary motor cortex based on cortico-motoneuronal cells. Proc. Natl. Acad. Sci. U.S.A. 106, 918-923. doi: 10.1073/pnas.0808362106

Rearick, M. P., Johnston, J. A., and Slobounov, S. M. (2001). Feedback-dependent modulation of isometric force control: an EEG study in visuomotor integration. Cogn. Brain Res. 12, 117-130. doi: 10.1016/S0926-6410(01)00040-4

Schaffelhofer, S., Agudelo-Toro, A., and Scherberger, H. (2015). Decoding a wide range of hand configurations from macaque motor, premotor, and parietal cortices. J. Neurosci. 35, 1068-1081. doi: 10.1523/JNEUROSCI.3594-14.2015 
Taylor, D. M., Helms Tillery, S. I., and Schwartz, A. B. (2002). Direct cortical control of 3D neuroprosthetic devices. Science 296, 1829-1832. doi: 10.1126/science.1070291

Thumser, Z. C., Slifkin, A. B., Beckler, D. T., and Marasco, P. D. (2018). Fitts' law in the control of isometric grip force with naturalistic targets. Front. Psychol. 9:560. doi: 10.3389/fpsyg.2018.00560

Velliste, M., Perel, S., Spalding, M. C., Whitford, A. S., and Schwartz, A. B. (2008). Cortical control of a prosthetic arm for self-feeding. Nature 453, 1098-1101. doi: 10.1038/nature06996

Wang, K., Wang, Z., Guo, Y., He, F., Qi, H., Xu, M., et al. (2017). A brain-computer interface driven by imagining different force loads on a single hand: an online feasibility study. J. Neuroeng. Rehabil. 14, 1-10. doi: 10.1186/s12984-017-0307-1

Wodlinger, B., Downey, J. E., Tyler-Kabara, E. C., Schwartz, A. B., Boninger, M. L., and Collinger, J. L. (2014). Ten-dimensional anthropomorphic arm control in a human brain-machine interface: difficulties, solutions, and limitations. J. Neural Eng. 12:16011. doi: 10.1088/1741-2560/12/1/016011

Conflict of Interest Statement: The authors declare that the research was conducted in the absence of any commercial or financial relationships that could be construed as a potential conflict of interest.

Copyright (c) 2018 Downey, Weiss, Flesher, Thumser, Marasco, Boninger, Gaunt and Collinger. This is an open-access article distributed under the terms of the Creative Commons Attribution License (CC BY). The use, distribution or reproduction in other forums is permitted, provided the original author(s) and the copyright owner(s) are credited and that the original publication in this journal is cited, in accordance with accepted academic practice. No use, distribution or reproduction is permitted which does not comply with these terms. 